\title{
New 2-in-1 Polyelectrolyte Step-by-Step Film Buildup without Solution Alternation: From PEDOT-PSS to Polyelectrolyte Complexes
}

\author{
Christine de Saint-Aubin, ${ }^{\dagger}$ Joseph Hemmerlé, $^{\ddagger}$ Fouzia Boulmedais, ${ }^{\S} \|$ Marie-France Vallat, ${ }^{\dagger}$ \\ Michel Nardin, ${ }^{\dagger}$ and Pierre Schaaf*, ${ }^{\S}, \|$ \\ ${ }^{\dagger}$ Centre National de la Recherche Scientifique, Institut de Science des Matériaux de Mulhouse, LRC 7228 - UHA, 15 rue Jean \\ Starcky, BP 2488, 68057 Mulhouse cedex, France \\ ${ }^{\ddagger}$ Institut National de la Santé et de la Recherche Médicale, UMR 977, 11, rue Humann, 67085 Strasbourg cedex, France \\ ${ }^{\S}$ Centre National de la Recherche Scientifique, Institut Charles Sadron, UPR 22, 23 rue du Loess, BP 84047, 67034 Strasbourg cedex \\ 2, France \\ "International Center for Frontier Research in Chemistry (icFRC), 8 allée Gaspard Monge, 67083 Strasbourg, France
}

\section{Supporting Information}

\begin{abstract}
Although never emphasized and increasingly used in organic electronics, PEDOT-PSS (poly(3,4-ethylenedioxythiophene)poly(styrene sulfonate)) layer-by-layer (lbl) film construction violates the alternation of polyanion and polycation rule stated as a prerequisit for a step-by-step film buildup. To demonstrate that this alternation is not always necessary, we studied the step-by-step construction of films using a single solution containing polycation/polyanion complexes. We investigated four different systems: PEDOT-PSS, bPEI-PSS (branched poly(ethylene imine)-poly(sodium 4-styrene sulfonate)), PDADMA-PSS (poly(diallyl dimethyl ammonium)-PSS), and PAH-PSS (poly-

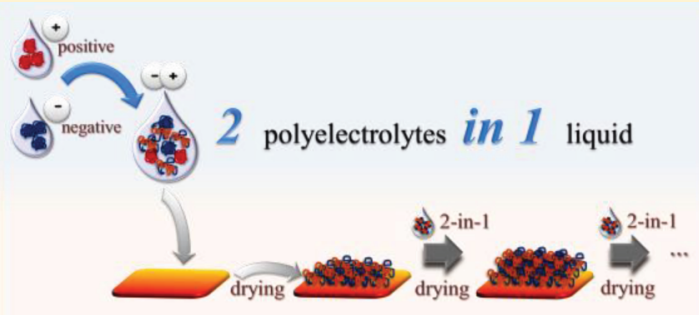
(allylamine hydrochloride)-PSS). The film buildup obtained by spin-coating or dipping-and-drying process was monitored by ellipsometry, UV-vis-NIR spectrophotometry, and quartz-crystal microbalance. The surface morphology of the films was characterized by atomic force microscopy in tapping mode. After an initial transient regime, the different films have a linear buildup with the number of deposition steps. It appears that, when the particles composed of polyanion-polycation complex and complex aggregates in solution are more or less liquid (case of PEDOT-PSS and bPEI-PSS), our method leads to smooth films (roughness on the order of 1-2 nm). On the other hand, when these complexes are more or less solid particles (case of PDADMA-PSS and PAH-PSS), the resulting films are much rougher (typically $10 \mathrm{~nm}$ ). Polycation/polyanion molar ratios in monomer unit of the liquid, rinsing, and drying steps are key parameters governing the film buildup process with an optimal polycation/polyanion molar ratio leading to the fastest film growth. This new and general lbl method, designated as 2-in-1 method, allows obtaining regular and controlled film buildup with a single liquid containing polyelectrolyte complexes and opens a new route for surface functionalization with polyelectrolytes.
\end{abstract}

\section{INTRODUCTION}

Among different surface functionalization methods, the layerby-layer (lbl) method, introduced independently by Iler and Kirkland in 1965-1966, ${ }^{1,2}$ was extended to polyelectrolyte multilayers by Decher in the 1990s. ${ }^{3}$ This method has become one of the most versatile and popular tools for surface functionalization, ${ }^{4}$ enabling covering surfaces of all types and shapes with films of well-defined composition and tailored thicknesses.

Polyelectrolyte multilayers result from the alternate deposition of polyanions and polycations onto a substrate. ${ }^{3}$ The versatility of the $\mathrm{lbl}$ method lies in the large variety of the compounds that can be deposited: polyelectrolytes, ${ }^{3}$ proteins, ${ }^{5}$ polysaccharides, ${ }^{6}$ clays, $^{7}$ and more generally inorganic nanoparticles, ${ }^{8,9}$ and recently polyelectrolyte complexes. ${ }^{10-20}$ Thus, depending on the pursued aim, surfaces with well-defined properties can be obtained: conductive surfaces by using multilayers containing for example PEDOT-PSS (poly $(3,4-$ ethylenedioxythiophene)-poly(styrenesulfonate) $)^{21-34}$ (Table 1 ), anti-inflammatory ones by using suitable peptides ${ }^{35}$ or mechanically responsive ones by using polyelectrolytes depositing on silicon ${ }^{36}$ to mention only some striking examples.

It is well-accepted that polyelectrolyte multilayer buildup processes require the alternation of a positive and negative excess charge (charge overcompensation) after each deposition step. Cohesion of polyelectrolyte multilayers is thus essentially based on electrostatic interactions even if other types of intermolecular forces can also come into play. ${ }^{37}$ Yet, at least one of the deposited species must be of polymeric nature. On one hand, the bond multiplicity accounts for the enthalpic

Received: March 26, 2012

Revised: $\quad$ May 17, 2012

Published: May 17, 2012 
Table 1. Chemical Formulae of the Polyelectrolytes Used in the Present Work and Monomer Unit Molar Masses Used to Describe Them; Schematic Representation of the PEDOTPSS Complex

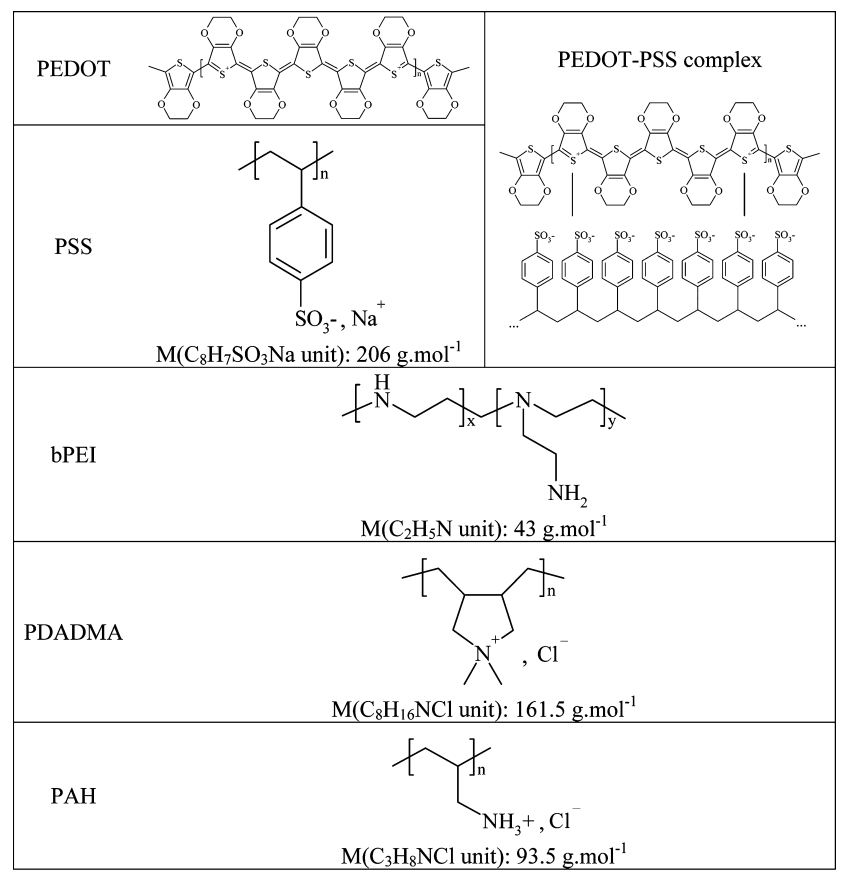

stability of the obtained multilayered structures. On the other hand, the entropic stability is rooted in the structural disorder increase coming from the release after multilayer construction of most of the counterions initially bound to the deposited polyelectrolytes. $^{38}$

If the alternate $\mathrm{lbl}$ method offers plenty of surface functionalization possibilities, it is not devoid, however, of drawbacks inherent in its very nature. For example, if one has decided to deposit a species chosen for a specific property, a complementary species has to be assigned to it, and most often, this association dilutes this desired property. This is typically what happened for electrically conducting multilayer films in which the globally negative PEDOT-PSS was associated with a generally nonconducting polycation ${ }^{21-34}$ to satisfy to the charge alternation rule. Yet, this generally takes place at the expense of the conductivity, due to the adjunction of a nonconductive polycation. ${ }^{24-26}$ PEDOT-PSS is an interesting example, since it is a zwitterionic yet globally negatively charged compound widely used in photovoltaics, OLEDs (organic lightemitting diods), or photodiods. Surprisingly, it was also reported that such devices can be obtained from aqueous suspensions of the negatively charged PEDOT-PSS deposited step-by-step on the fit substrate by spin-coating, ${ }^{39-41}$ inkjet printing, ${ }^{42-44}$ or spraying ${ }^{45}$ processes without any alternation with a positively charged species. This thus seems to violate the alternation rule that has been postulated as a prerequisite for film buildup by the multilayer community. This aspect, as well as the required conditions for such a buildup, have, however, not been addressed and not even mentioned in those papers. This observation raises the question of the necessity of a charge alternation in the step-by-step film buildup based on electrostatic interactions. In other words, could a new $\mathrm{lbl}$ method, designated as 2-in-1 method, be invented which would implicate the deposition of a single zwitterionic species or a polyanion/ polycation complex liquid? This new 2 -in-1 method would offer the advantages of the alternate $\mathrm{lbl}$ method-now qualified as traditional-but at the same time, it would overcome the traditional one's limits, mainly the necessary charge alternation.

To answer these questions, we first went back to the PEDOT-PSS example to confirm and extend some of the results mentioned in the organic electronics literature. Then, we generalized these observations with bPEI-PSS (branched poly(ethyleneimine)-poly(sodium 4-styrenesulfonate)), PDADMA-PSS (poly(diallyldimethylammonium)-PSS), and PAH-PSS (poly(allylaminehydrochloride)-PSS) complexes to demonstrate the general feasibility of the 2 -in-1 method. These complexes were chosen because these polyelectrolytes belong to the most employed ones in traditional alternate lbl method. The film construction was followed by ellipsometry, UV-visNIR spectrophotometry, and QCM (quartz crystal microbalance). The obtained 2-in-1 films were also characterized by atomic force microscopy in tapping-mode (AFM).

\section{EXPERIMENTAL SECTION}

Materials. Poly(3,4-ethylenedioxythiophene)-poly(styrene sulfonate) (PEDOT-PSS) $1.3 \mathrm{wt} \%$ dispersion in $\mathrm{H}_{2} \mathrm{O}$ (conductive grade), poly(sodium 4-styrene sulfonate) (PSS) ( $M_{\mathrm{w}} \sim 70000$ $\left.\mathrm{g} \cdot \mathrm{mol}^{-1}\right)$, branched poly(ethylene imine) (bPEI) solution $\left(M_{\mathrm{w}}\right.$ $\sim 750000 \mathrm{~g} \cdot \mathrm{mol}^{-1} ; 50 \%(\mathrm{w} / \mathrm{v})$ in $\left.\mathrm{H}_{2} \mathrm{O}\right)$, poly(diallyldimethylammonium chloride) (PDADMA) solution $\left(M_{\mathrm{w}}\right.$ 100 000-200 $000 \mathrm{~g} \cdot \mathrm{mol}^{-1} ; 20$ wt $\%$ in $\mathrm{H}_{2} \mathrm{O}$ ), and poly(allylamine hydrochloride) (PAH) $\left(M_{\mathrm{w}} \sim 56000 \mathrm{~g} \cdot \mathrm{mol}^{-1}\right)$ were purchased from Aldrich. All the products were used as received. The formulas of the different products are shown in Table 1 . Water purified via reverse osmosis (Millipore Elix system, min. $5 \mathrm{M} \Omega . \mathrm{cm}$, max. $15 \mathrm{M} \Omega . \mathrm{cm}$ ) was the solvent used for all except QCM experiments. For the QCM experiments, Milli-Q water (Milli-Q Plus system, Millipore, Billerica, MA) was used. Phosphorus n-doped [100] oriented silicon wafers were obtained from Mat Technology (Morangis, France). Quartz slides were bought from Hellma (Paris, France). Gold-coated QCM (quartz crystal microbalance) sensors from Q-Sense AB (Gothenburg, Sweden) were used for QCM experiments.

Preparation of Substrates. Two kinds of substrates were used, silicon wafers and quartz slides. Silicon-oxide-on-silicon substrates were prepared by cutting silicon wafers to approximately $5 \mathrm{~mm} \times 10$ $\mathrm{mm}$ pieces, rinsing them with cyclohexane and drying them under a nitrogen stream. This cleaning was sufficient because of the buffering role of the precursor layers (see below) directly deposited on the substrate. Quartz slides were rinsed with water and ethanol and dried under a nitrogen stream.

Preparation of Spin-Coated Films. A TP6000 with CT60 controller (Karl Suss, Saint Jeoire, France) spin-coater was used for the preparation of the spin-coated films.

(bPEI-al-PSS) ${ }_{2}$ Precursor Layers. If necessary (see below), underlying precursor layers built by alternate depositions of bPEI and PSS aqueous solutions were applied onto the silicon or quartz substrate. Therefore, a $0.6 \mathrm{mg} / \mathrm{mL}$ ultrasonicated aqueous solution of the positively charged bPEI $\left(\mathrm{p} K_{\mathrm{a}}=8.2-8.3^{46}\right)$ was manually spread over the whole surface of the negatively charged substrate and immediately spin-coated during $30 \mathrm{~s}$ at $5000 \mathrm{rpm}$, with an initial acceleration of $5000 \mathrm{rpm} / \mathrm{s}$. A $0.7 \mathrm{mg} / \mathrm{mL}$ solution of the negatively charged PSS $\left(\mathrm{p} K_{\mathrm{a}}\right.$ $=1^{47}$ ) was then deposited in the same manner, without any intermediate rinsing or further drying. The whole procedure was repeated to obtain precursor layers designated as (bPEI-al-PSS) 2 " al" meaning that the two substances, bPEI and PSS, were alternatively deposited. In other words, (bPEI-al-PSS) ${ }_{2}$ represents a system where bPEI, PSS, bPEI, and finally PSS were successively deposited.

(bPEI-al-PSS) ${ }_{2}$ (PEDOT-Cx-PSS) ${ }_{n}$ System. A 0.25 wt \% PEDOT-PSS dispersion, obtained by diluting the commercial one, was spread over the whole surface of a (bPEI-al-PSS $)_{2}$ covered substrate and spincoated in the same manner as for the precursor layers. The process was repeated $n$ times to reach a system designated as (PEDOT-cx-PSS) ${ }_{n}$ : " $c x$ " meaning that the PEDOT-PSS complex between the two 
substances PEDOT and PSS was deposited $n$ times. Because both positive and negative species were simultaneously present in the unique mixture used for the film buildup, we named this step-by-step method the 2-in-1 method. Following this nomenclature, the entire deposited system, including the precursor one, was designated as (bPEI-al-PSS) $)_{2}$ (PEDOT-cx-PSS) $n, n$ referring to the deposition number. We intentionally avoid calling $n$ the "layer number", because $n$ does not necessarily describe the deposition of a complete monolayer of matter.

(bPEI-al-PSS) ${ }_{2}$ (bPEI-cx-PSS) ${ }_{n}$ System. Mixtures of bPEI complexed by PSS were obtained by adding ultrasonicated bPEI aqueous solutions of desired concentrations to known NaPSS masses diluted in smallest water volumes. After adjusting to the appropriate volume, the mixtures were thoroughly shaken. The concentrations in monomer of each polymer were in the range $10^{-2}-10^{-3} \mathrm{~mol} / \mathrm{L}$ in the final solution of adjusted bPEI to PSS molar ratio in monomer unit (the molar ratios are calculated from the mass concentration of each polyelectrolyte divided by the mass of corresponding monomer unit as given in Table 1). Care had to be taken during the preparation of theses mixtures, as we observed that reversing the order of mixing hindered the subsequent film buildup. To obtain a film of a given composition, one such mixture was spread over the entire surface of a (bPEI-al-PSS) ${ }_{2}$ covered substrate and spin-coated in the same manner as for the precursor layers. This was repeated $n$ times. Following our nomenclature, the entire deposited system, including the precursor one, was thus designated as (bPEI-al-PSS $)_{2}$ (bPEI-cx-PSS)

$(b P E I-a l-P S S)_{2} \quad$ (PDADMA-cX-PSS) ${ }_{n}$ and (bPEI-al-PSS) $)_{2}$ (PAH-CX$P S S)_{n}$ System. Mixtures containing PDADMA (respectively, PAH) complexed by PSS were prepared by mixing together PDADMA (respectively, PAH) and PSS aqueous solutions at a desired concentration (concentrations in monomer of each polymer in the range $\left.10^{-3}-10^{-4} \mathrm{~mol} / \mathrm{L}\right)$. The (bPEI-al-PSS) ${ }_{2}$ (PDADMA-cx-PSS) ${ }_{n}$ (respectively, (bPEI-al-PSS) ${ }_{2}$ (PAH-cx-PSS $)_{n}$ ) systems were then obtained analogously to the (bPEI-al-PSS) ${ }_{2}$ (bPEI-cx-PSS) ${ }_{n}$ systems.

Preparation of Films Followed by Quartz Crystal Microbalance (QCM). Prior to use, Au-covered quartz crystals and QCME4 apparatus (Q-Sense) were rinsed successively with water, Hellmanex $2 \%$, water, $0.1 \mathrm{~mol} / \mathrm{L}$ aqueous $\mathrm{HCl}$, and water, and finally dried with compressed air.

(bPEI-al-PSS) ${ }_{2}$ (PEDOT-cx-PSS) ${ }_{1}$ System. During the experiment, the quartz crystal was first put into contact with water during $3 \mathrm{~min}$. Then, the (bPEI-al-PSS) 2 precursor layers were adsorbed on the crystal. Therefore, $0.6 \mathrm{~mL}$ of one polyelectrolyte at a flow rate of 1 $\mathrm{mL} / \mathrm{min}$ was flown over the crystal and then left at rest for $5 \mathrm{~min}$. Afterward, the sample was rinsed with $0.6 \mathrm{~mL}$ Milli-Q water at a flow rate of $1 \mathrm{~mL} / \mathrm{min}$, then left at rest for another $5 \mathrm{~min}$. These durations were sufficient to reach constant values for the frequency changes and hence constant amounts of the deposited polyelectrolytes. The same procedure was repeated to reach the whole (bPEI-al-PSS) 2 precursor layers. The attempts to deposit a 0.25 wt \% PEDOT-PSS dispersion (obtained as explained earlier) onto these precursor layers are described in the Results and Discussion section.

(PEI-Cx-PSS) ${ }_{1}$ and (PDADMA-Cx-PSS) ${ }_{1}$ Systems. After contact with water during $3 \mathrm{~min}$, the clean quartz crystal was put into contact with the PEI-cx-PSS or PDADMA-cx-PSS liquid systems prepared as described above.

Ellipsometry. Null ellipsometric measurements were performed using the Multiskop (Optrel GBR, Berlin, Germany), operating at 532 $\mathrm{nm}$ at a $70^{\circ}$ angle of incidence, with a beam spot of $0.6 \mathrm{~mm}$. Measurements were made at six different spots on each sample, the data being acquired by the Optrel software Multi. The data were treated with the Optrel software Elli. The model used to calculate the thickness $t$ and the real refractive index $N$ of the studied polyelectrolyte system was composed of four parallel layers: a silicon layer with a refractive index of $N=4.1501$ (real) and $k=0$ (imaginary), a silicon oxide layer with $N=1.4607$ and $k=0$ and a thickness determined for each new sample, the studied polyelectrolyte system layer, and finally an air layer with $N=1.0000$ and $k=0$.

UV-vis-NIR Spectroscopy. UV-vis-NIR absorption of the samples was investigated using Lambda 750 (Perkin-Elmer, Shelton,
USA) or Evolution 220 (Thermo Scientific, Madison, USA) spectrometers. The PEDOT-PSS films were deposited on quartz slides precoated with (PEI-alt-PSS) ${ }_{2}$ that were taken as reference. The PDADMA-cx $x$-PSS liquid systems were analyzed in glass cuvettes; the cuvettes filled with Millipore water were taken as reference.

Quartz Crystal Microbalance. The QCM-D (Quartz Crystal Microbalance with dissipation-monitoring) experiments were performed on a QCM-E4 (Q-Sense) apparatus. The fundamental resonance frequency as well as the third, fifth, and seventh harmonics (respectively, near 5, 15, 25, and $35 \mathrm{MHz}$ ) of the gold-coated quartz supporting the multilayer system were acquired at $25{ }^{\circ} \mathrm{C}$, using $\mathrm{Q}$ soft 401 2.0.1.288 software. As the Sauerbrey relationship ${ }^{48}$ did not hold for our viscoelastic deposited films, the frequency shifts were directly used, without further mass calculations: a negative frequency shift was associated with a mass increase, a positive shift with a mass decrease. The dissipation factor $D_{\mathrm{i}}$, which is a qualitative measure of the viscoelasticity of the film, was calculated by the Q-soft401 software: the higher $D_{\mathrm{i}}$ was, the more viscous the film.

Atomic Force Microscopy. Tapping-mode AFM images in air were acquired on Multimode Scanning Probe Microscopes Nanoscope III and IV (Digital Instruments Veeco Technology group, Plainview, USA) to study the morphology, rms (root-mean-square) roughness $R_{\mathrm{q}}$ and thickness of the constructed films. Nanoworld Arrow-NC silicon probes (radius of curvature $<10 \mathrm{~nm}$ ) were used for imaging the samples. Imaging was done at a fixed scan rate of $1 \mathrm{~Hz}(4 \mathrm{~Hz}$ for PEIPSS samples) with a resolution of $512 \times 512$ pixels. The obtained images were processed with both Nanoscope 6.13r1 (Digital Instruments, Veeco) and WS $x M 3.0$ (Nanotec Electronica S.L.) software. ${ }^{49}$ The rms roughness $R_{\mathrm{q}}$ was calculated by the Nanoscope software from height images $(1 \mu \mathrm{m} \times 1 \mu \mathrm{m})$ after prior automatic planefit and manual third-order flattening treatment to remove any tilt and bow artifacts. To measure the thickness of the deposited films, the samples were deliberately scratched with plastic micropipet tips and the thickness was obtained with the section tool of the Nanoscope software. Each thickness value is the result of 5 height measurements along the scratch.

Dynamic Light Scattering. DLS (dynamic light scattering) of backscattered light was measured at $25{ }^{\circ} \mathrm{C}$ using Vasco particle size analyzer (Cordouan Technologies, Pessac, France) operating with a laser at $657 \mathrm{~nm}$. For each solution containing complexes to be tested, three samples were taken, and at least five measurements were made on each sample, the scattered light intensity fluctuation data being acquired by Cordouan Technologies NanoQ software. From these data, the Brownian diffusion coefficient was calculated by the software, using a Padé-Laplace inversion algorithm. The mean hydrodynamic diameters by the number of polymer complexes and complex aggregates dispersed or solubilized in water were obtained via the Stokes-Einstein law. For these calculations, a refractive index of 1.33 and a dynamic viscosity of $0.894 \mathrm{mPa}$.s were taken for the water solvent, and a real refractive index of 1.590 was taken for the polymer particles.

\section{RESULTS AND DISCUSSION}

Nanometric PEDOT-PSS Films Obtained by the 2-in-1 Method. PEDOT-PSS is a complex between PEDOT (Table 1), an oligomer of 6 to 18 structural units ${ }^{50}$ and PSS (Table 1), a strong polyelectrolyte $\left(\mathrm{p} K_{\mathrm{a}}=1\right)^{47}$ of 100 to 1000 structural units, depending on the molar mass. ${ }^{51}$ Part of PEDOT's structural unit in the PEDOT-PSS is positively charged because of an electron loss during the synthesis of PEDOT by EDOT oxidation. ${ }^{51}$ PSS is a negatively charged polymer in aqueous solution, as the great majority of its sulfonic acid groups are deprotonated in sulfonates $\mathrm{SO}_{3}{ }^{-}$at all $\mathrm{pH}$ values. The interaction between PEDOT and PSS in the PEDOT-PSS complex is thus electrostatic (Table 1). In the PEDOT-PSS complex, many PEDOT oligomers bind to the long chain of PSS. $^{50}$ The commercial aqueous suspension is composed of PEDOT-PSS chains $(\sim 5 \%)$ swollen by water $(\sim 95 \%)$ forming 
gel particles (diameter of about $40 \mathrm{~nm}$ ), ${ }^{50}$ suspended in water. The monomeric ratio of PEDOT to PSS of the commercial aqueous suspension used was $0.8 .^{52}$ As the negative charges of PSS are in excess with respect to the positive charges of PEDOT, the PEDOT-PSS complex is globally negatively charged. The nonagglomeration of the PEDOT-PSS gel particles comes from the electrostatic repulsion between the excess sulfonates pointing out of each of these particles. Besides, deposited PEDOT-PSS gel particles show a core richer in PEDOT than the outer shell, the latter being enriched in PSS. $^{53,54}$

Our work starts with the successful buildup of PEDOT-PSS films by successively depositing PEDOT-PSS on itself using a spin-coater. Figure 1 (upper curve) shows the evolution of the

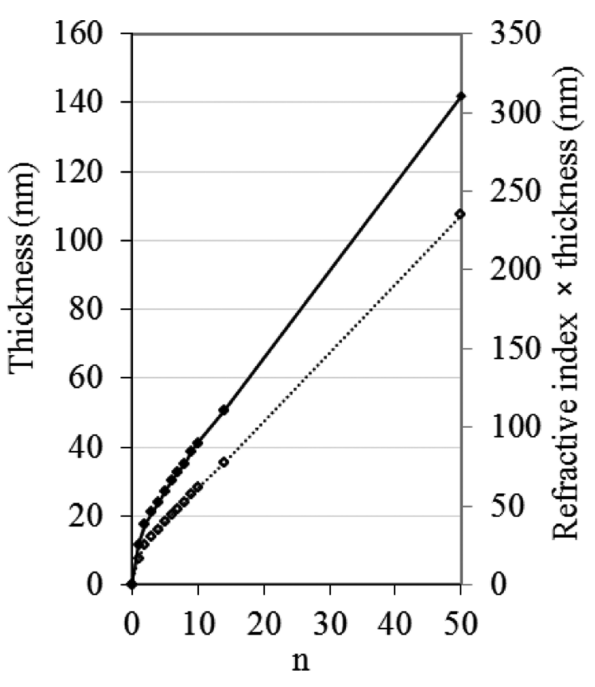

Figure 1. Film thickness (filled diamond-shaped symbols) and product of the film refractive index and thickness (empty diamond-shaped symbols), obtained by ellipsometry, of a spin-coated (PEDOT-cxPSS $)_{n}$ film built on a $\mathrm{Si} / \mathrm{SiO}_{2} /(\text { bPEI-al-PSS })_{2}$ wafer, as a function of the deposition number $n$. Note that the curves serve only as a guide for the eye. thickness, determined by ellipsometry, of a (PEDOT-cx-PSS) film built on $\mathrm{Si} / \mathrm{SiO}_{2} /$ (bPEI-al-PSS $)_{2}$ as a function of the number of deposition steps $n$. The film thickness increases steadily with the increase of the deposition number. Furthermore, the growth becomes perfectly linear after an initial transient regime of approximately five deposition steps (linear regression coefficient of 0.9996 calculated on the linear part of the curve). The film growth rate in the linear regime is equal to $2 \mathrm{~nm}$ /deposition. Exactly the same linear buildup, with the same growth rate, was also observed with one layer of bPEI as a precursor layer instead of (bPEI-al-PSS) ${ }_{2}$ (data not shown). After the initial transient regime, the growth process is independent of the substrate. It should, however, be noted that at least one bPEI precursor layer is necessary for the film buildup on the silicon wafer, as shown by the failure of PEDOT-PSS deposition on the bare substrate. Moreover, after one year storage under ambient conditions, the film thickness remained unchanged proving the stability of the film.

The product of the film thickness and refractive index, directly proportional to the deposited mass, follows the same behavior as the thickness versus the deposition number (Figure 1 lower curve). After the initial transient regime, the deposited mass increases linearly as the deposition number increases.

Complementarily, Figure 2 illustrates how the UV-vis-NIR spectrum, measured between 190 and $1000 \mathrm{~nm}$, of a spincoated (PEDOT-cx-PSS) $)_{n}$ film built on precoated (bPEI-alPSS $)_{2}$ quartz substrate evolves with successive deposition steps. PEDOT's polaronic (peak around $840 \mathrm{~nm}$ ) and bipolaronic (band extending until the short wavelength infrared) absorptions $^{30}$ as well as peaks at 192 and $224 \mathrm{~nm}$ characteristic of styrene sulfonate increase with the deposition number $n$. More precisely, the absorbances, characteristic of both PEDOT and PSS, increase linearly (linear regression coefficient 0.9939 at $192 \mathrm{~nm}, 0.9867$ at $224 \mathrm{~nm}$, and 0.9952 at $910 \mathrm{~nm}$ ) after an initial transient regime of approximately five deposition steps (insets in Figure 2). This confirms the linear increase of the mass of deposited PEDOT and PSS.

Let us now describe the surface morphology of spin-coated PEDOT-PSS films obtained with the 2-in-1 method. Figure 3a gives the 3D-height AFM image of a $\mathrm{Si} / \mathrm{SiO}_{2} /(\mathrm{bPEI}-\text { al-PSS })_{2}$

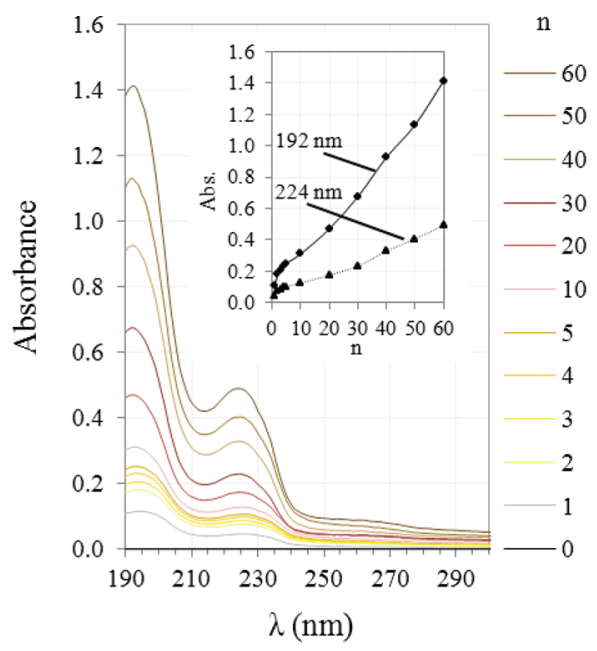

(a)

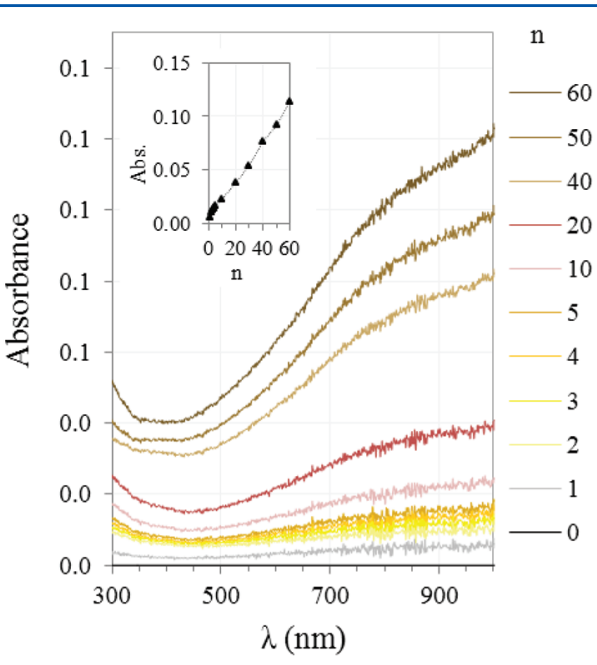

(b)

Figure 2. UV-vis-NIR absorbance spectrum (a) from 190 to $300 \mathrm{~nm}$ and (b) from 300 to $1000 \mathrm{~nm}$, of a spin-coated (PEDOT-cx-PSS) film, built on a (bPEI-al-PSS) $)_{2}$ precoated quartz substrate, at different number of deposition $n$. Insets: evolution of the film absorbance (a) at 192 and $224 \mathrm{~nm}$ and (b) at $910 \mathrm{~nm}$, plotted versus the deposition number $n$. 
a

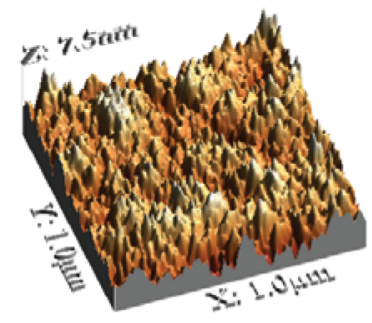

b
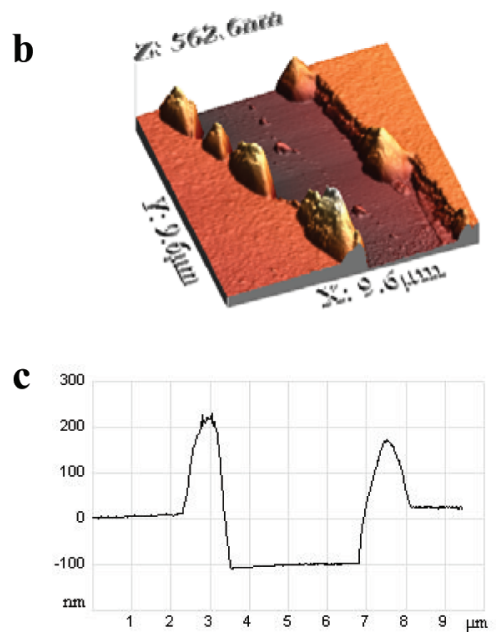

Figure 3. Tapping-mode AFM images of a spin-coated (PEDOT-cxPSS $)_{50}$ film built on a precoated $\mathrm{Si} / \mathrm{SiO}_{2} /(\text { bPEI-al-PSS })_{2}$ wafer: (a) 3D-height of a $1 \mu \mathrm{m} \times 1 \mu \mathrm{m}$ surface of the film, (b) 3D-height of a 9.6 $\mu \mathrm{m} \times 9.6 \mu \mathrm{m}$ surface, and (c) the first-order flattened section of the height-image parallel to the $z$-axis of the scratched film.

(PEDOT-cx-PSS $)_{50}$ film obtained by AFM in tapping mode. The granular structure observed is identical to the one obtained by several authors after a unique deposition of PEDOT-PSS with or without thermal annealing. ${ }^{55-59}$ (PEDOT-cx-PSS) films are very smooth with a nanometric rms roughness of $0.8 \mathrm{~nm}$. Moreover, these structure and roughness values remain constant all along the buildup from $n=5$ to 50 (data not shown). The tuning of the film thickness keeping the roughness constant is valuable, since very smooth PEDOT-PSS films are needed in photovoltaic or OLED applications. ${ }^{45,59,60}$

Another way to look at the surface morphology of 2-in-1 PEDOT-cx-PSS films on another scale is given by Figure $3 b, c$, mapping a deliberate scratch made on the film. The section extracted from this mapping depicts as well the very small roughness of the 2-in-1 film. The scratch clearly emerges from a very smooth film which can be seen on both of its sides (obviously, abstraction has to be made of the clear piling up on both sides of the scratch, due to accumulation there of the matter extracted from the scratch). Additionally, the film thickness obtained from this section is $130 \pm 20 \mathrm{~nm}$. This result agrees, within the experimental errors, with the value of $112 \pm$ $16 \mathrm{~nm}$ obtained for the same film by ellipsometry.

A careful analysis of the AFM images reveals that the films appear as composed of flat particles (1-3 nm in thickness) and of lateral dimension of the order of $41 \pm 9 \mathrm{~nm}$ (see SI part I). This seems to indicate that the films form by sintering of the PEDOT-PSS gel-like particles initially present in solution, the sintering being due to the interdiffusion of the PEDOT-PSS chains.
To further investigate the 2-in-1 method applied to PEDOTPSS, the influence of different parameters on the construction was studied. First of all, the results obtained by dipping-anddrying deposition of PEDOT-PSS were strictly analogous to the ones obtained by spin-coating: it is thus not the centrifuge force exerted during the spin-coating deposition that can explain the successful 2-in-1 PEDOT-PSS buildup (see SI part II-A). By contrast, attempts to make successive depositions of PEDOT-PSS within the QCM-apparatus failed. After a first PEDOT-PSS deposition, the construction nearly stopped (see SI part III): only a slight mass increase was observed, which was attributed to the completion of the PEDOT-PSS monolayer. What is missing in the QCM-apparatus deposition process with respect to the successful dipping-and-drying process is the drying step. It seems therefore reasonable to state that the drying step allows a restructuring of the upper part of the multilayer film that renders the surface more compatible for an additional deposition of PEDOT-PSS particles. Indeed, reversible structural changes have been observed after drying. ${ }^{61}$ PEDOT-PSS is known for being hygroscopic due to the hydrophilic property of PSS. ${ }^{61-64}$

In a further experiment (Figure 4), each spin-coating deposition of the PEDOT-PSS suspension was followed by

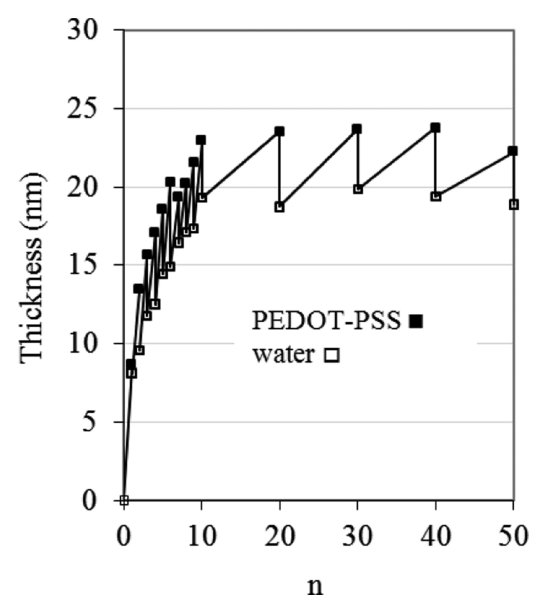

Figure 4. Film thickness, measured by ellipsometry, of a spin-coated (PEDOT-c $x$-PSS $)_{x}$ film built on a precoated $\mathrm{Si} / \mathrm{SiO}_{2} /(\text { bPEI-al-PSS })_{2}$ wafer as a function of deposition number $n$. The film was built by alternating spin-coating of PEDOT-PSS solutions (filled squares) and of water rinsing solution (empty squares). Note that the curve serves only as a guide for the eye.

spin-coating pure water in the same conditions. This rinsing step seems to hinder the film construction. The thickness of the film stabilizes at $20 \mathrm{~nm}$ after the first 10 deposition steps followed by $3 \mathrm{~nm}$ of thickness increase at each PEDOT-PSS deposition immediately lost at the rinsing step. During the first 10 deposition steps, the surface is probably covered with a monolayer of strongly attached PEDOT-PSS gel particles. The final thickness of $20 \mathrm{~nm}$ is reached when the coverage of the surface is total. This is supported by the fact that the diameter of the PEDOT-PSS gel particles is about $40 \pm 10 \mathrm{~nm}$ (as measured by dynamic light scattering). The difference between both dimensions could come from the squashing of gel particles due to the adsorption on the underlying $\mathrm{Si} / \mathrm{SiO}_{2} /$ (bPEI-alPSS $)_{2}$ layers. Similar flattening of block copolymer micelles upon adsorption was reported in the literature. ${ }^{65-67}$ After the monolayer formation when PEDOT-PSS is further deposited, 
matter attaches very loosely. During the rinsing step, the loosely adsorbed, water-soluble ${ }^{68}$ PEDOT-PSS moves back into the aqueous solution. The next deposition and rinsing steps occur exactly in the same manner, and therefore, no film construction can take place over the monolayer. In related experiments, the solubility of 2-in-1 PEDOT-PSS films was verified by immersion of the films in pure Millipore water for one hour. After this immersion, the film thicknesses were reduced to more than $90 \%$ of their initial value and the blue color characteristic of the films totally disappeared.

To conclude this first part of the paper, the 2 -in- 1 method applied to PEDOT-PSS is able to provide films of tailored nanothickness ( $2 \mathrm{~nm}$ per deposition step) with the same surface morphology ${ }^{55-59}$ and at least the same low roughness $^{57,59}$ as the thicker films obtained after a unique deposition. Until now, nanofilms of PEDOT-PSS were obtained by the lbl method by alternating the negative PEDOT-PSS with a positively charged polyelectrolyte or with positively charged particles. $^{21-34}$ Our work demonstrates that the sole PEDOTPSS can be successfully deposited in nanometrically controlled thickness, without the need of charge alternation, thanks to the zwitterionic nature of PEDOT-PSS. The properties of PEDOTPSS films, obtained by the 2 -in- 1 method, i.e., conductivity, transparency, stability, and low cost, are not, in essence, changed by the adjunction of a second species, as there is no second species. Although analogous multilayered PEDOT-PSS films were already obtained by a few teams, no systematic study of the layer-by-layer buildup was done. Only in rare cases, linear quasi step-by-step buildup was followed in a range of higher thicknesses (from 60 to $1450 \mathrm{~nm}$ per deposition step). ${ }^{39,41,44} \mathrm{In}$ the other studies, the linear growth was brought up or supposed. ${ }^{40,42,43,45}$ Last but not least, to our knowledge, the question never arose of the possibility for such a deposition of PEDOT-PSS on PEDOT-PSS.

This result must be striking and proves that the $\mathrm{lbl}$ method has to be reconsidered in terms of charge alternation. It seems that this charge alternation is not necessary if local zwitterionic charge distributions are available. It becomes obvious that the PEDOT-PSS case should not be an isolated case. And indeed, we now generalize the 2 -in- 1 method by using other polyelectrolyte complexes.

Generalization of the 2-in-1 Method on Different Polyelectrolyte Complexes. Until the present work, films based on polyelectrolyte complexes were obtained by the traditional lbl method, i.e., by alternate deposition of the polyelectrolyte complex solution and another solution containing oppositely charged polyelectrolytes or polyelectrolyte complexes. ${ }^{10-20}$ Contrary to the traditional $\mathrm{lbl}$ method, the 2in-1 method of deposition is based on successive depositions of the same solution containing the polyelectrolyte complexes and complex-aggregates.

We followed the buildup of different polyelectrolyte complexes films, on (PEI-al-PSS $)_{2}$ precursor layers, by the 2in-1 method using a spin-coater. Complexes aqueous solution of bPEI-PSS, PDADMA-PSS, and PAH-PSS were, respectively, prepared at a molar polycation to polyanion ratio in monomer of 50, 0.24 , and 2 (the molar ratios are calculated from the mass concentration of each polyelectrolyte divided by the mass of corresponding monomer unit as given in Table 1). In Figure 5, the buildup of bPEI-cx-PSS, PDADMA-cx-PSS, and PAH-cxPSS films was monitored by ellipsometry. The polyelectrolytes of these systems were chosen for their diversity in type (strong or weak), in molecular mass (chain length), and in shape
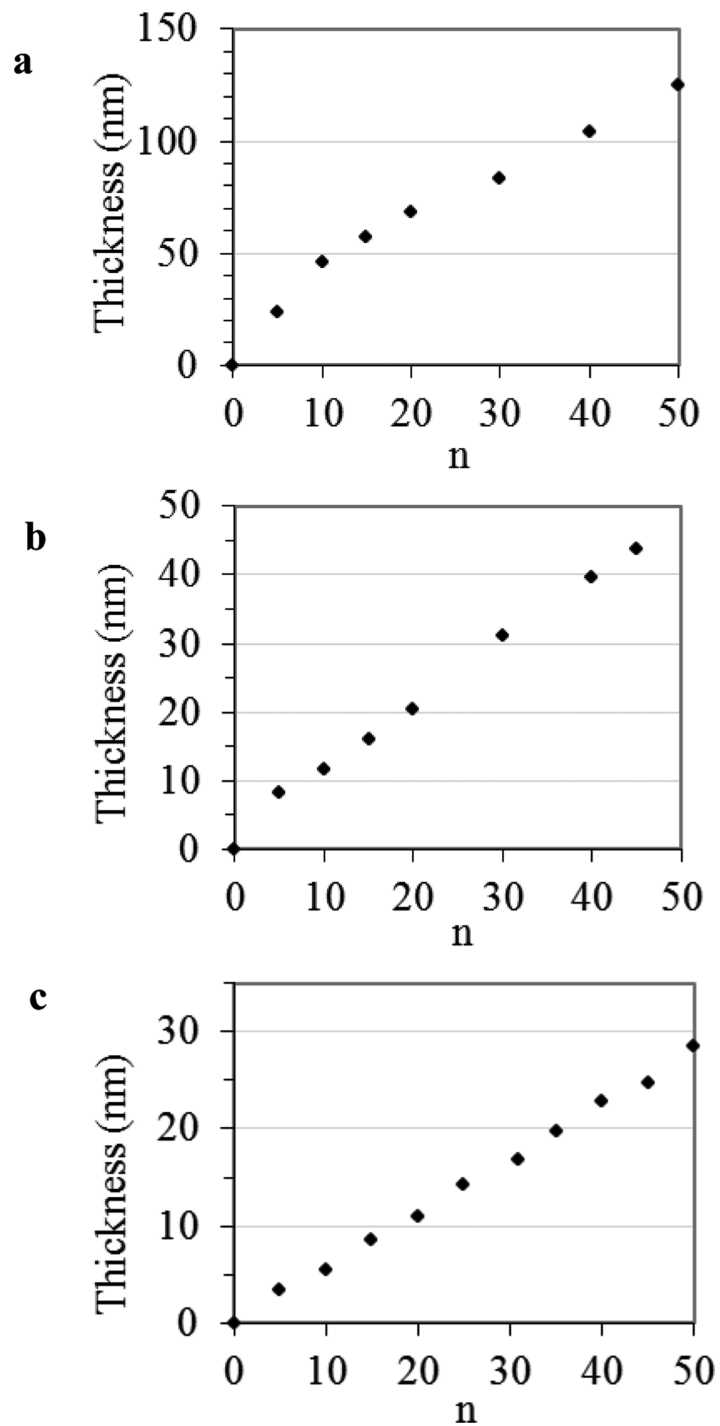

Figure 5. Thickness, measured by ellipsometry, as a function of the deposition number $n$ of (a) (bPEI-cx-PSS) ${ }_{n}$ film built with bPEI-PSS complex aqueous solution at a molar bPEI/PSS ratio of 50 in monomer, (b) (PDADMA-cx-PSS) film built with PDADMA-PSS at a molar PDADMA/PSS ratio of 0.24 and (c) (PAH-cx-PSS) film built with PAH-PSS at a molar PAH/PSS ratio of 2 . The films were built applying the 2 -in- 1 method by spin-coating on precoated $\mathrm{Si} / \mathrm{SiO}_{2} /$ (bPEI-al-PSS $)_{2}$ wafer substrates.

(linear or branched). They also belong to the most studied polyelectrolytes in the lbl community. In our experiments, these polyelectrolytes were used in aqueous medium without ionic strength or $\mathrm{pH}$ adjustments. All the polyelectrolyte systems show linear growth of film thickness with an increase of the deposition number after an initial transient regime (linear regression coefficient of 0.997, 0.999, and 0.998, respectively, for bPEI-cx-PSS, PDADMA-cx-PSS, and PAH-cx-PSS). Further experiments on bPEI-PSS and PDADMA-PSS complex solutions showed a similar film buildup without a precursor layer on the wafer substrate; this was not observed for the PEDOT-cx-PSS previously.

Morphology of bPEI-cx-PSS, PDADMA-cx-PSS, and PAH$c x$-PSS films was monitored by AFM in tapping mode (Figure 6). In all cases, the film covers the entire substrate. The films seem granular, but depending on the polyelectrolyte complexes used, the size of the grains is different. The rms roughness is 


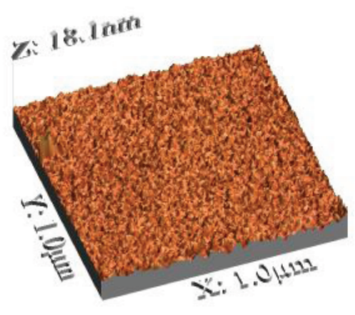

c

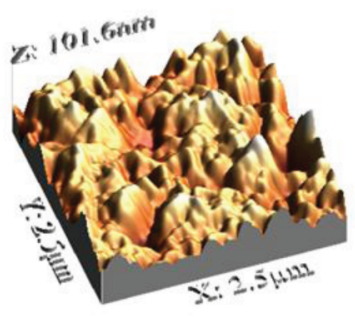

e

\section{$(\mathrm{PAH}-c x-\mathrm{PSS})_{50}$}

b

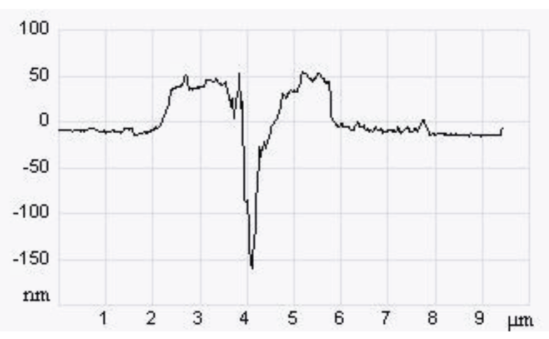

d

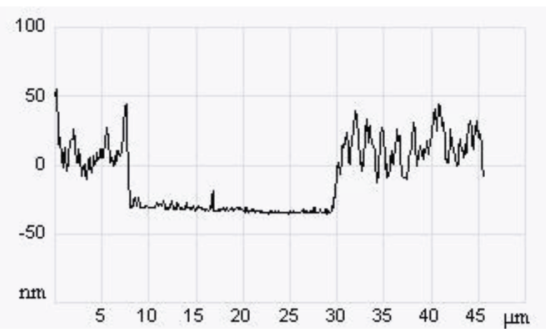

f

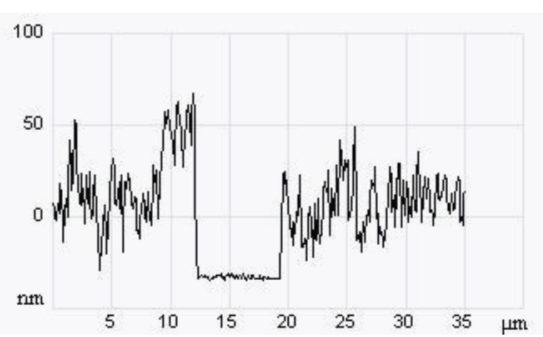

Figure 6. Tapping-mode AFM 3D-height images $(1 \mu \mathrm{m} \times 1 \mu \mathrm{m})$ and the respective section of the height-image after scratching of spin-coated (a) (bPEI-cx-PSS $_{50}$ film built with bPEI-PSS complexes aqueous solution at a molar bPEI/PSS ratio of 50 in monomer, (c) (PDADMA-cx-PSS) 50 film built with PDADMA-PSS at a PDADMA/PSS molar ratio of 0.24 and (e) (PAH-cx-PSS $)_{50}$ film built with PAH-PSS at a PAH/PSS molar ratio of 2. The films were built applying the 2 -in- 1 method by spin-coating on precoated $\mathrm{Si} / \mathrm{SiO}_{2} /(\mathrm{bPEI} \text {-al-PSS })_{2}$ wafer substrates.

Table 2. Mean Size of Particle and Particle Aggregate of Polycation-PSS Complex ${ }^{a}$

$\begin{array}{ccccc}\text { polycation-PSS complex } & \begin{array}{c}\text { liquid: particle mean size } \\ (\mathrm{nm})(*)\end{array} & \begin{array}{c}\text { film: lateral particle mean size } \\ (\mathrm{nm})(* *)\end{array} & \begin{array}{c}\text { liquid: particle aggregate mean } \\ \text { size }(\mathrm{nm})(*)\end{array} & \begin{array}{c}\text { film: lateral particle aggregate mean } \\ \text { size }(\mathrm{nm})(* *)\end{array} \\ \text { bPEI-PSS (bPEI/PSS }=50) & 31 \pm 4 & 40 \pm 9 & - & - \\ \text { PDADMA-PSS (PDADMA/ } & 230 \pm 60 & 270 \pm 50 & 1000 \pm 400 & 800 \pm 400 \\ \text { PSS = 0.24) } & 160 \pm 40 & 90 \pm 20 & 600 \pm 200 & 240 \pm 80 \\ \text { PAH-PSS (PAH/PSS = 2) } & & & \end{array}$

${ }^{a}(*)$ In the liquid solution or suspension, measured by DLS; $(* *)$ in the film built on precoated $\mathrm{Si} / \mathrm{SiO}_{2} /(\mathrm{PEI} \text {-alt-PSS })_{2}$ wafer substrate, measured by tapping mode AFM. The polycation/PSS ratios given between brackets are the molar polycation to PSS monomer ratios. Size uncertainties are expressed as standard deviations (calculated over 15 values in the liquid samples and over 10 values in the film samples).

extending from ca. $10 \mathrm{~nm}$ for PDADMA-cx-PSS and PAH- $c x$ PSS films to less $0.5 \mathrm{~nm}$ for the bPEI-cx-PSS films. Interestingly, these surface morphologies differ from the ones obtained for traditional alternate films of the same composition (see SI part IV). So, using the 2-in-1 method, different nanotextures can be tailored from different complexes, which is valuable, since some applications like sensors need high exchange surfaces, while others prefer lower ones.

As for the PEDOT-PSS case, when carefully analyzing the different AFM images, it appears that the films are composed of small particles or particle aggregates that are more or less interpenetrated (see SI part I). The lateral size of these particles or particle aggregates correlates fairly well with the mean size of the particles measured by dynamic light scattering in solution (Table 2).

In the case of bPEI- $c x$-PSS, the thickness of the particles is on the order of 1-2 nm, whereas for PDADMA-cx-PSS and PAH$c x$-PSS, it is on the order of $10-30 \mathrm{~nm}$. The difference in thickness between bPEI-cx-PSS and the two other systems may be due to the fact that PEI is a branched polyelectrolyte so that the complexes it forms with PSS are much less dense than those between a linear polycation and a linear polyanion. The same argument may also explain the difference in morphology between the bPEI-cx-PSS film whose roughness is small $(0.5$ $\mathrm{nm}$ for a film of $150 \mathrm{~nm}$ thickness) compared to that of the two other systems (ca. $7 \mathrm{~nm}$ for films of $50 \mathrm{~nm}$ thickness). Indeed, the fact that bPEI is branched does not allow for a tight interaction between bPEI and PSS so that the film should behave more or less like a liquid. This is further supported by the softness experienced during tapping-mode AFM analysis (see self-healing behavior of the scratches like in Figure $6 \mathrm{~b}$ and hardly avoidable indentation during scanning like in SI part I.B). This somewhat liquid behavior is then similar to what was observed for the PEDOT-PSS case. On the contrary, for linear polyanions and polycations, where the interactions are strong, as is expected for PSS-PAH and to a smaller extent for 
PDADMA-PSS, the mobility of the polyelectrolytes should be more reduced and the small particles composing the films should behave more or less like small solid particles.

To gain deeper insight in the 2-in-1 method, the influence of different parameters was studied. First, the polycation/ polyanion molar monomer unit ratio (designated as polycation/polyanion ratio) introduced in the liquid was varied for the PDADMA/PSS and bPEI/PSS systems. Figure 7a summarizes

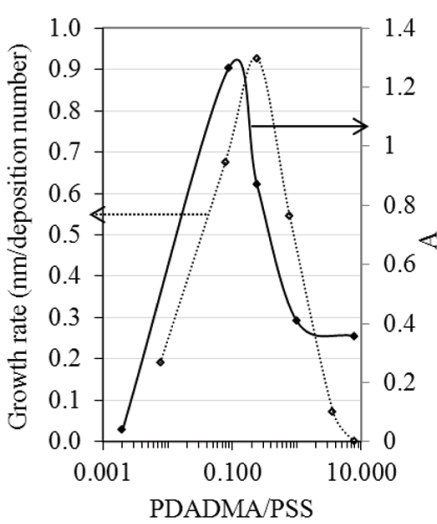

(a)

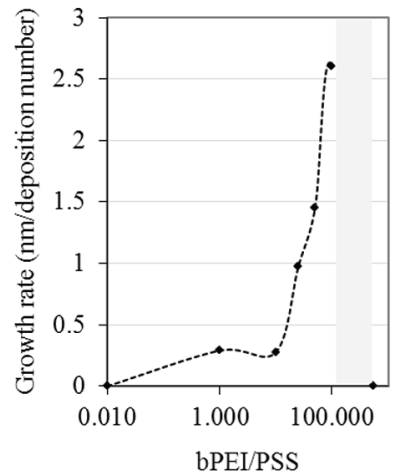

(b)
Figure 7. (a) Evolution (empty diamond-shaped symbols) of the PDADMA-cx-PSS film growth rate, measured by ellipsometry and (filled diamond-shaped symbols) of the PDADMA/PSS liquid turbidity A, measured at $1 \mathrm{~h}$ by UV-vis spectroscopy at $500 \mathrm{~nm}$ as a function of the PDADMA/PSS ratio initially introduced in the complex liquid. The films were built applying the 2 -in- 1 method by spin-coating on precoated $\mathrm{Si} / \mathrm{SiO}_{2} /(\mathrm{bPEI}-a l \text {-PSS })_{2}$ wafer substrates. (b) Evolution of bPEI-cx-PSS film growth rate, measured by ellipsometry, as a function of the bPEI/PSS ratio initially introduced in the liquid. In the shaded region, a hardly reproducible and nonregular film buildup occurs. The films were built applying the 2-in1 method by spin-coating on precoated $\mathrm{Si} / \mathrm{SiO}_{2} /(\text { bPEI-al-PSS })_{2}$ wafer substrates. Note that the curves serve only as a guide for the eye.

the film growth rate (in $\mathrm{nm} /$ deposition number) of PDADMA$c x$-PSS films obtained at different initial polycation/polyanion ratio. The growth rate was calculated in the linear growth regime (a growth rate equaling zero meaning that the film could not be constructed). The bell-shaped curve obtained indicates that the PDADMA/PSS ratio has a great influence on the film construction, with a maximum growth rate of $0.9 \mathrm{~nm} /$ deposition number obtained at PDADMA/PSS ratio of about 0.2 . For smaller or higher ratios, the growth rate is diminished, and the film no longer builds for ratio over approximately 10 .

A similar influence of the bPEI/PSS ratio was found on the bPEI- $c x$-PSS film growth rate (Figure $7 b$ ). For overly small bPEI/PSS ratios (from 0.010 to 10 ), the film hardly builds up at all. At higher ratio between 10 and 100, a linear buildup is obtained with an increase of growth rate with the bPEI/PSS ratio (with a maximum growth rate of $2.6 \mathrm{~nm} /$ deposition number). If the bPEI/PSS ratio exceeds 100, no regular but rather an abrupt buildup is observed: the film thickness jumps to a high thickness of approximately $180 \mathrm{~nm}$ within less than 10 deposition steps and then stagnates or even diminishes. At a ratio of 540, no film buildup is observed at all. For a linear and controllable 2 -in-1 bPEI-cx-PSS film construction, it is thus advisible to maintain the bPEI/PSS ratio between 10 and 100 .

To better understand the origin of the polycation/polyanion ratio influence on the 2-in-1 film buildup, we quantified the turbidity of the PDADMA/PSS liquid used for the different buildups. The turbidity allows measurement of the presence of nonsoluble complexes, which belong to a new phase and thus diffuse incoming light. Figure $7 \mathrm{a}$ also plots the turbidity of the spin-coated liquid, measured by UV-vis spectrophotometric at $500 \mathrm{~nm}$ (a wavelength where neither PDADMA nor PSS absorb) as a function of the PDADMA/PSS ratio. The absorbance curve coincides within experimental error with the growth rate curve (Figure 7a), indicating a strong correlation between the structure of the liquid used for the film construction and the structure of the film itself. Hence, the presence of insoluble PDADMA-cx-PSS complexes in the deposited solution allows film buildup by spin-coating using the 2 -in- 1 method. In the case of the bPEI-cx-PSS system, the 2-in1 method can be applied even if no visually detectable turbidity is observed for the entire tested ratio domain. Yet, this does not imply that no polyanion/polycation complexes form in solution, only that they are of too small size. In the case of bPEI-cx-PSS, they are on the order of $30 \mathrm{~nm}$ as measured by dynamic light scattering. Consequently, the phase ${ }^{69}$ or turbidity diagrams $^{70}$ that have already been established for many polyelectrolyte complexes may predict well the possibility of film buildup using the 2 -in- 1 method. This should accelerate the development of its use in the sense that, where coacervation has been detected, the 2-in-1 method might be applied with a good probability.

The dipping-and-drying process was compared to the spincoating process (see SI part II). No bPEI-cx-PSS films can be obtained with the dipping-and-drying process, whereas PDADMA- $c x$-PSS films are successfully deposited by this process but with a lower growth rate. Hence, the deposition process can affect the success of the 2 -in- 1 method. In our spincoating process, the solution is deposited in large excess on the whole surface of the substrate and spinning is started. The liquid is then first removed by the centrifuge force until the liquid film thickness reaches a critical size, which is on the order of a few micrometers. ${ }^{71}$ Once this critical thickness has been reached, the solution is entirely removed by evaporation of the solvent (water in our case) due to spinning. We never observed any film breakage during the whole procedure, and we ended with a dry film. When the spin-coating was performed in a humid atmosphere (closing the spin-coater), the film construction was much slower, indicating the importance of the drying step. By contrast, in the dipping-and-drying process, the excess material is first removed by drainage. The excess solution is then removed by a strong nitrogen stream so that not only the solvent, but also the complexes are evacuated, leading eventually to smaller film thicknesses. Yet, even in this case drying is absolutely necessary for the film to build up. For both bPEI-cx-PSS and PDADMA-cx-PSS systems, the film did not construct in the QCM apparatus where neither drying nor spinning take place (see SI part III) confirming the key role of drying. Intermediate rinsing steps affects the 2 -in- 1 film buildup (see SI part V). On one hand, the bPEI- $c x$-PSS film builds up with a loss of construction linearity. On the other hand, the PDADMA-cx-PSS film builds up with a slower growth rate. Furthermore and contrary to the PEDOT-cx-PSS case, when 2in-1 films of bPEI-cx-PSS with a bPEI/PSS ratio of 50 or PDADMA-cx-PSS with a PDADMA/PSS ratio of 0.24 or PAH$c x$-PSS with a PAH/PSS ratio of 2 are totally immersed in pure Millipore water for one hour, their thickness decreases only in a limited way $(8 \%, 17 \%$, and $5 \%$ thickness reduction, respectively, for films obtained after 50 depositions). This demonstrates the good stability of these 2 -in- 1 films in water. 
Knowing the key roles of the polycation/polyanion ratio and the drying and rinsing steps, we can rationalize the success of the 2-in-1 method. During the deposition, which occurs under kinetic control (see, for example, the influence of the order of introduction of PEI and PSS in the Experimental Section or that of the deposition process-spin-coating vs dipping-anddrying), the complexes and the complex aggregates present in the deposited liquid attach to the substrate by electrostatic interactions between the surface and the local charges present in the complexes. During the drying step, through spin-coating or $\mathrm{N}_{2}$-stream, a reorganization of the deposited polyelectrolytes occurs that enables further complex or complex aggregate deposition. Without this reorganization, the film cannot build up. If the exact nature of this reorganization remains unclear, some phenomena can, however, be hypothesized. The reorganization could consist of a sole polyelectrolyte chain reorientation, which would produce a surface charge distribution more compatible with the next complexes to be deposited. It could also involve more vigorous global polyelectrolyte chain movements, permitted by the shear force due to the air movement and/or by the centrifugal force in the spin-coater, which here again would render charge compatibility with the next complexes to be deposited more satisfactory.

\section{CONCLUSION}

We have introduced a new 2-in-1 method of deposition of polyelectrolyte film consisting of successive depositions of the same liquid containing the polyelectrolyte complexes and complex-aggregates. This method allows control of the thickness at the nanometric scale to obtain thin polyelectrolytes films (<100 $\mathrm{nm}$ approx.). By analogy with the traditional alternate $\mathrm{lbl}$ method, parameters like ionic strength ${ }^{72}$ or $\mathrm{pH}^{73}$ should most probably influence the 2 -in-1 film buildup, enabling, for example, higher growth rates, if needed. Moreover, the surface morphologies of the 2 -in-1 films can be different from the ones obtained with the traditional alternate $\mathrm{lbl}$ method for the same polyelectrolyte pair and with the same composition. It seems to appear that when the particles composed of polyanion/polycation complex aggregates in solution are more or less liquid (case of PEDOT-PSS and bPEI-PSS) the 2-in-1 method leads to smooth films. On the other hand, when these particles are more or less solid (case of PDADMA-PSS and PAH-PSS), the resulting films are much rougher. Yet, this conclusion needs to be further investigated. The 2-in-1 method offers new potentialities for the implementation of $\mathrm{lbl}$ film. From an industrial point of view, it simplifies the design of the equipment needed: it only takes a sole fluid circuit for a sole solution as well as the control of the deposition parameters, which do not need to be doubled. This new method is also promising to the numerous PEDOT-PSS users in the organic electronics domain, as it opens reproducible nanometric thickness control of very smooth films. Finally and above all, the 2 -in- 1 method enables the deposition of a unique complex, which is fundamental if it is the only species to be deposited and/or if this complex cannot be separated into its constituents. As so, the method outstrips the traditional one by avoiding the necessity of alternate deposition of positively and negatively charged species. This might be particularly valuable for the deposition of biological complexes formed by charged subunits, as polyelectrolyte/protein and polyelectrolyte/DNA complexes. Hence, the 2 -in-1 method will take the advantage of the high amount of knowledge from the traditional alternate $\mathrm{lbl}$ method, but at the same time, it will be able to go beyond the bonds of tradition.

\section{ASSOCIATED CONTENT}

\section{S Supporting Information}

Experimental data on lateral grain size of deposited complexes, studies of the effects of the deposition process (spin-coating vs dipping-and-drying, QCM film buildup), of the drying, and of the rinsing on the 2-in-1 method and comparison on the morphologies of the 2 -in- 1 vs alternate samples. This material is available free of charge via the Internet at http://pubs.acs.org.

\section{AUTHOR INFORMATION}

\section{Corresponding Author}

*Phone: +33 (0)3 884140 12. E-mail: schaaf@ics.u-strasbg.fr. Notes

The authors declare no competing financial interest.

\section{ACKNOWLEDGMENTS}

CdSA was supported by the French "Ministère de l'Éducation nationale, de la Jeunesse et de la Vie associative" and by the French "Agence Nationale de la Recherche" (ANR-10-BLAN0818 "Biostretch"). Thanks to Mohammad El Hajj Hassan for his contribution to UV-vis spectroscopic data collection.

\section{REFERENCES}

(1) Iler, R. K. Multilayers of colloidal particles. J. Colloid Interface Sci. 1966, 21, 569-594.

(2) Kirkland, J. J. Porous Thin-Layer Modified Glass Bead Supports for Gas Liquid Chromatography. Anal. Chem. 1965, 37, 1458-1461.

(3) Decher, G. Fuzzy Nanoassemblies: Toward Layered Polymeric Multicomposites. Science 1997, 277, 1232-1237.

(4) Hammond, P. T. Engineering materials layer-by-layer: Challenges and opportunities in multilayer assembly. AIChE J. 2011, 57, 29282940.

(5) Lvov, Y.; Ariga, K.; Kunitake, T. Layer-by-layer assembly of alternate protein polyion ultrathin films. Chem. Lett. 1994, 2323-2326.

(6) Picart, C.; Schneider, A.; Etienne, O.; Mutterer, J.; Schaaf, P.; Egles, C.; Jessel, N.; Voegel, J.-C. Controlled Degradability of Polysaccharide Multilayer Films In Vitro and In Vivo. Adv. Funct. Mater. 2005, 15, 1771-1780.

(7) Kleinfeld, E. R; Ferguson, G. S. Stepwise Formation of Multilayered Nanostructural Films from Macromolecular Precursors. Science 1994, 265, 370-373.

(8) Kotov, N. A.; Dekany, I.; Fendler, J. H. Layer-by-Layer SelfAssembly of Polyelectrolyte-Semiconductor Nanoparticle Composite Films. J. Phys. Chem. 1995, 99, 13065-13069.

(9) Lvov, Y.; Ariga, K.; Ichinose, I.; Kunitake, T. Formation of Ultrathin Multilayer and Hydrated Gel from Montmorillonite and Linear Polycations. Langmuir 1996, 12, 3038-3044.

(10) Schuetz, P.; Caruso, F. Multilayer thin films based on polyelectrolyte-complex nanoparticles. Colloids Surf., A 2002, 207, $33-40$.

(11) Cho, J.; Quinn, J. F.; Caruso, F. Fabrication of Polyelectrolyte Multilayer Films Comprising Nanoblended Layers. J. Am. Chem. Soc. 2004, 126, 2270-2271.

(12) Quinn, A.; Such, G. K.; Quinn, J. F.; Caruso, F. Polyelectrolyte Blend Multilayers: A Versatile Route to Engineering Interfaces and Films. Adv. Funct. Mater. 2008, 18, 17-26.

(13) Zhang, L.; Li, Y.; Sun, J.; Shen, J. Mechanically Stable Antireflection and Antifogging Coatings Fabricated by the Layer-byLayer Deposition Process and Postcalcination. Langmuir 2008, 24, 10851-10857.

(14) Guo, Y.; Geng, W.; Sun, J. Layer-by-Layer Deposition of Polyelectrolyte-Polyelectrolyte Complexes for Multilayer Film Fabrication. Langmuir 2009, 25, 1004-1010. 
(15) Liu, X.; Dai, B.; Zhou, L.; Sun, J. Polymeric complexes as building blocks for rapid fabrication of layer-by-layer assembled multilayer films and their application as superhydrophobic coatings. J. Mater. Chem. 2009, 19, 497-504.

(16) Zhang, L.; Sun, J. Layer-by-layer deposition of polyelectrolyte complexes for the fabrication of foam coatings with high loading capacity. Chem. Commun. 2009, 3901-3903.

(17) Zhao, Q.; Qian, J.; An, Q.; Du, B. Speedy fabrication of freestanding layer-by-layer multilayer films by using polyelectrolyte complex particles as building blocks. J. Mater. Chem. 2009, 19, $8448-8455$

(18) Zhang, L.; Sun, J. Layer-by-Layer Codeposition of Polyelectrolyte Complexes and Free Polyelectrolytes for the Fabrication of Polymeric Coatings. Macromolecules 2010, 43, 2413-2420.

(19) Porcel, C. H.; Schlenoff, J. B. Compact Polyelectrolyte Complexes: "Saloplastic" Candidates for Biomaterials. Biomacromolecules 2009, 10, 2968-2975.

(20) Zeng, G.; Gao, J.; Chen, S.; Chen, H.; Wang, Z.; Zhang, X. Combining Hydrogen-Bonding Complexation in Solution and Hydrogen-Bonding-Directed Layer-by-Layer Assembly for the Controlled Loading of a Small Organic Molecule into Multilayer Films. Langmuir 2007, 23, 11631-11636.

(21) Tang, Z.; Donohoe, S. T.; Robinson, J. M.; Chiarelli, P. A.; Wang, H.-L. Film formation, surface character, and relative density for electrochromic PEI/(PSS:PEDOT) multilayered thin films. Polymer 2005, 46, 9043-9052.

(22) Agarwal, M.; Lvov, Y.; Varahramyan, K. Conductive wood microfibres for smart paper through layer-by-layer nanocoating. Nanotechnology 2006, 17, 5319.

(23) Zheng, Z.; McDonald, J.; Khillan, R.; Su, Y.; Shutava, T.; Grozdits, G.; Lvov, Y. M. Layer-by-Layer Nanocoating of Lignocellulose Fibers for Enhanced Paper Properties. J. Nanosci. Nanotechnol. 2006, 6, 624-632.

(24) Dawidczyk, T. J.; Walton, M. D.; Jang, W.-S.; Grunlan, J. C. Layer-by-Layer Assembly of UV-Resistant Poly(3,4-ethylenedioxythiophene) Thin Films. Langmuir 2008, 24, 8314-8318.

(25) Cutler, C. A.; Bouguettaya, M.; Reynolds, J. R. PEDOT Polyelectrolyte Based Electrochromic Films via Electrostatic Adsorption. Adv. Mater. 2002, 14, 684-688.

(26) Smith, R. R.; Smith, A. P.; Stricker, J. T.; Taylor, B. E.; Durstock, M. F. Layer-by-Layer Assembly of Poly(3,4ethylenedioxythiophene):Poly(styrenesulfonate). Macromolecules 2006, 39, 6071-6074.

(27) Wistrand, I.; Lingström, R.; Wågberg, L. Preparation of electrically conducting cellulose fibres utilizing polyelectrolyte multilayers of poly(3,4-ethylenedioxythiophene):poly(styrene sulphonate) and poly(allyl amine). Eur. Polym. J. 2007, 43, 4075-4091.

(28) Nah, Y.-C.; Choi, W. S.; Kim, D.-Y. Preparation and electrochromic properties of spin self-assembled polyelectrolyte multilayer films composed of PEDOT:PSS and PAH. Sol. Energy Mater. Sol. Cells 2008, 92, 1547-1551.

(29) Wakizaka, D.; Fushimi, T.; Ohkita, H.; Ito, S. Hole transport in conducting ultrathin films of PEDOT/PSS prepared by layer-by-layer deposition technique. Polymer 2004, 45, 8561-8565.

(30) DeLongchamp, D.; Hammond, P. T. Layer-by-Layer Assembly of PEDOT/Polyaniline Electrochromic Devices. Adv. Mater. 2001, 13, 1455-1459.

(31) DeLongchamp, D. M.; Kastantin, M.; Hammond, P. T. HighContrast Electrochromism from Layer-By-Layer Polymer Films. Chem. Mater. 2003, 15, 1575-1586.

(32) Khillan, R.; Ghan, R.; Dasaka, R.; Su, Y.; Lvov, Y.; Varahramyan, $\mathrm{K}$. Layer-by-layer nanoarchitecture of ultrathin films assembled of PEDOT-PSS and PPy to act as hole transport layer in polymer light emitting diodes and polymer transistors. In 4th IEEE International Conference on Polymers and Adhesives in Microlelectronics and Photonics; 2004; pp 225-229.

(33) Ho, P.; Kim, J.; Burroughes, J.; Becker, H.; Li, S.; Brown, T.; Cacialli, F.; Friend, R. Molecular-scale interface engineering for polymer light-emitting diodes. Nature 2000, 404, 481-484.
(34) Sakai, N.; Prasad, G. K.; Ebina, Y.; Takada, K.; Sasaki, T. Layerby-Layer Assembled $\mathrm{TiO} 2$ Nanoparticle/PEDOT-PSS Composite Films for Switching of Electric Conductivity in Response to Ultraviolet and Visible Light. Chem. Mater. 2006, 18, 3596-3598.

(35) Schultz, P.; Vautier, D.; Richert, L.; Jessel, N.; Haikel, Y.; Schaaf, P.; Voegel, J.-C.; Ogier, J.; Debry, C. Polyelectrolyte multilayers functionalized by a synthetic analogue of an anti-inflammatory peptide, $\alpha$-MSH, for coating a tracheal prosthesis. Biomaterials 2005, 26, 26212630.

(36) Hemmerlé, J.; Roucoules, V.; Fleith, G.; Nardin, M.; Ball, V.; Lavalle, P.; Marie, P.; Voegel, J.-C.; Schaaf, P. Mechanically Responsive Films of Variable Hydrophobicity Made of Polyelectrolyte Multilayers. Langmuir 2005, 21, 10328-10331.

(37) Zhang, X.; Chen, H.; Zhang, H. Layer-by-layer assembly: from conventional to unconventional methods. Chem. Commun. 2007, $1395-1405$

(38) Bertrand, P.; Jonas, A.; Laschewsky, A.; Legras, R. Ultrathin polymer coatings by complexation of polyelectrolytes at interfaces: suitable materials, structure and properties. Macromol. Rapid Commun. 2000, 21, 319-348.

(39) Ham, H. T.; Choi, Y. S.; Chee, M. G.; Cha, M. H.; Chung, I. J. PEDOT-PSS/singlewall carbon nanotubes composites. Polym. Eng. Sci. 2008, 48, 1-10.

(40) Colsmann, A.; Stenzel, F.; Balthasar, G.; Do, H.; Lemmer, U. Plasma patterning of Poly(3,4-ethylenedioxythiophene):Poly(styrenesulfonate) anodes for efficient polymer solar cells. Thin Solid Films 2009, 517, 1750-1752.

(41) Kwon, I. W.; Son, H. J.; Kim, W. Y.; Lee, Y. S.; Lee, H. C. Thermistor behavior of PEDOT:PSS thin film. Synth. Met. 2009, 159, 1174-1177.

(42) Ballarin, B.; Fraleoni-Morgera, A.; Frascaro, D.; Marazzita, S.; Piana, C.; Setti, L. Thermal inkjet microdeposition of PEDOT:PSS on ITO-coated glass and characterization of the obtained film. Synth. Met. 2004, 146, 201-205.

(43) Natori, A. Y.; Canestraro, C. D.; Roman, L. S.; Ceschin, A. M. Modification of the sheet resistance of ink jet printed polymer conducting films by changing the plastic substrate. Mater. Sci. Eng., B 2005, 122, 231-235.

(44) Ummartyotin, S.; Juntaro, J.; Wu, C.; Sain, M.; Manuspiya, H. Deposition of PEDOT: PSS Nanoparticles as a Conductive Microlayer Anode in OLEDs Device by Desktop Inkjet Printer. J. Nanomater. 2011, 2011.

(45) Tedde, S. F.; Kern, J.; Sterzl, T.; Fürst, J.; Lugli, P.; Hayden, O. Fully Spray Coated Organic Photodiodes. Nano Lett. 2009, 9, 980983.

(46) Choosakoonkriang, S.; Lobo, B. A.; Koe, G. S.; Koe, J. G.; Middaugh, C. R. Biophysical characterization of PEI/DNA complexes. J. Pharm. Sci. 2003, 92, 1710-1722.

(47) Lvov, Y.; Ariga, K.; Onda, M.; Ichinose, I.; Kunitake, T. A careful examination of the adsorption step in the alternate layer-by-layer assembly of linear polyanion and polycation. Colloids Surf., A 1999, 146, 337-346.

(48) Sauerbrey, G. Verwendung von Schwingquarzen zur Wägung dünner Schichten und zur Mikrowägung. Z. Phys. A-Hadron. Nucl. 1959, 155, 206-222.

(49) Horcas, I.; Fernandez, R.; Gomez-Rodriguez, J. M.; Colchero, J.; Gomez-Herrero, J.; Baro, A. M. WSXM: A software for scanning probe microscopy and a tool for nanotechnology. Rev. Sci. Instrum. 2007, 78, 013705-8.

(50) Kirchmeyer, S.; Reuter, K.; Simpson, J. C. Poly(3,4-Ethylenedioxythiophene)-Scientific Importance, Remarkable Properties, and Applications. In Handbook of Conducting Polymers, Skotheim, T. A., Reynolds, J., Eds.; CRC Press: Boca Raton, 2007; pp 10-1 to 1022.

(51) Krafft, W.; Jonas, F.; Muys, B.; Quintens, D. Antistatische Kunststoffteile. Patent EP 0564911 A2. 1993.

(52) Sigma-Aldrich. Product info \#483095. http://www.sigmaaldrich. com (accessed mars 14, 2012). 
(53) Greczynski, G.; Kugler, T.; Salaneck, W. R. Characterization of the PEDOT-PSS system by means of X-ray and ultraviolet photoelectron spectroscopy. Thin Solid Films 1999, 354, 129-135.

(54) Greczynski, G.; Kugler, T.; Keil, M.; Osikowicz, W.; Fahlman, M.; Salaneck, W. R. Photoelectron spectroscopy of thin films of PEDOT-PSS conjugated polymer blend: a mini-review and some new results. J. Electron. Spectrosc. 2001, 121, 1-17.

(55) Kemerink, M.; Timpanaro, S.; de Kok, M. M.; Meulenkamp, E. A.; Touwslager, F. J. Three-Dimensional Inhomogeneities in PEDOT:PSS Films. J. Phys. Chem. B 2004, 108, 18820-18825.

(56) Snaith, H. J.; Kenrick, H.; Chiesa, M.; Friend, R. H. Morphological and electronic consequences of modifications to the polymer anode 'PEDOT:PSS'. Polymer 2005, 46, 2573-2578.

(57) Wang, S.-J.; Park, H.-H. Study of PEDOT:PSS-SnO2 nanocomposite film as an anode for polymer electronics. J. Electroceram. 2007, 18, 161-165.

(58) Nardes, A. M.; Janssen, R. A. J.; Kemerink, M. A Morphological Model for the Solvent-Enhanced Conductivity of PEDOT:PSS Thin Films. Adv. Funct. Mater. 2008, 18, 865-871.

(59) Steirer, K. X.; Berry, J. J.; Reese, M. O.; van Hest, M. F. A. M.; Miedaner, A.; Liberatore, M. W.; Collins, R. T.; Ginley, D. S. Ultrasonically sprayed and inkjet printed thin film electrodes for organic solar cells. Thin Solid Films 2009, 517, 2781-2786.

(60) Hoth, C. N.; Steim, R.; Schilinsky, P.; Choulis, S. A.; Tedde, S. F.; Hayden, O.; Brabec, C. J. Topographical and morphological aspects of spray coated organic photovoltaics. Org. Electron. 2009, 10, 587593.

(61) Nardes, A. M. On the conductivity of PEDOT:PSS thin films. Ph.D. Thesis, Technische Universiteit, Eindhoven, 2007.

(62) Huang, J.; Miller, P. F.; Wilson, J. S.; de Mello, A. J.; de Mello, J. C.; Bradley, D. D. C. Investigation of the Effects of Doping and PostDeposition Treatments on the Conductivity, Morphology, and Work Function of Poly(3,4-ethylenedioxythiophene)/Poly(styrene sulfonate) Films. Adv. Funct. Mater. 2005, 15, 290-296.

(63) Fehse, K.; Meerheim, R.; Walzer, K.; Leo, K.; Lovenich, W.; Elschner, A. Lifetime of organic light emitting diodes on polymer anodes. Appl. Phys. Lett. 2008, 93, 083303-3.

(64) Okuzaki, H.; Hosaka, K.; Suzuki, H.; Ito, T. Effect of temperature on humido-sensitive conducting polymer actuators. Sens. Actuator, A 2010, 157, 96-99.

(65) Talingting, M. R.; Ma, Y.; Simmons, C.; Webber, S. E. Adsorption of Cationic Polymer Micelles on Polyelectrolyte-Modified Surfaces. Langmuir 1999, 16, 862-865.

(66) Webber, G. B.; Wanless, E. J.; Bütün, V.; Armes, S. P.; Biggs, S. Self-Organized Monolayer Films of Stimulus-Responsive Micelles. Nano Lett. 2002, 2, 1307-1313.

(67) Gensel, J.; Betthausen, E.; Hasenohrl, C.; Trenkenschuh, K.; Hund, M.; Boulmedais, F.; Schaaf, P.; Muller, A. H. E.; Fery, A. Surface immobilized block copolymer micelles with switchable accessibility of hydrophobic pockets. Soft Matter 2011, 7, 11144-11153.

(68) Ghosh, S.; Inganäs, O. Conducting Polymer Hydrogels as 3D Electrodes: Applications for Supercapacitors. Adv. Mater. 1999, 11, 1214-1218.

(69) Sukhishvili, S. A.; Kharlampieva, E.; Izumrudov, V. Where Polyelectrolyte Multilayers and Polyelectrolyte Complexes Meet. Macromolecules 2006, 39, 8873-8881.

(70) Mjahed, H.; Voegel, J.-C.; Chassepot, A.; Senger, B.; Schaaf, P.; Boulmedais, F.; Ball, V. Turbidity diagrams of polyanion/polycation complexes in solution as a potential tool to predict the occurrence of polyelectrolyte multilayer deposition. J. Colloid Interface Sci. 2010, 346, 163-171.

(71) Merrill, M. H.; Sun, C. T. Fast, simple and efficient assembly of nanolayered materials and devices. Nanotechnology 2009, 20, 075606.

(72) Ladam, G.; Schaad, P.; Voegel, J. C.; Schaaf, P.; Decher, G.; Cuisinier, F. In Situ Determination of the Structural Properties of Initially Deposited Polyelectrolyte Multilayers. Langmuir 1999, 16, 1249-1255.
(73) Shiratori, S. S.; Rubner, M. F. pH-Dependent Thickness Behavior of Sequentially Adsorbed Layers of Weak Polyelectrolytes. Macromolecules 2000, 33, 4213-4219. 